PROCEEDINGS OF THE

AMERICAN MATHEMATICAL SOCIETY

Volume 129, Number 1, Pages 237-246

S 0002-9939(00)05753-1

Article electronically published on June 7, 2000

\title{
ON STABLE CURRENTS AND POSITIVELY CURVED HYPERSURFACES
}

\author{
YI-BING SHEN AND QUN HE \\ (Communicated by Jozef Dodziuk)
}

\begin{abstract}
We establish a nonexistence theorem for stable currents (or stable varifolds) in complete $\delta$-pinched hypersurfaces of a real space form with nonnegative constant sectional curvature. This is a partial positive answer to the well-known conjecture of Lawson and Simons.
\end{abstract}

\section{$\S 1$. INTRODUCTION}

A Riemannian manifold $M$ is said to be $\delta$-pinched for $0<\delta \leq 1$ if the sectional curvature $K_{M}$ of $M$ satisfies $\delta a \leq K_{M} \leq a$ everywhere for some positive number $a$. One may take $a=1$ usually. As a generalization of stable minimal submanifolds, a stable current is a rectifiable current which is a local minimum for the norm (or mass) functional. In their paper [LS] on stable currents, Lawson and Simons show that there are no stable currents (or, more generally, stable varifolds) on the Euclidean sphere, and propose the following

Conjecture. There are no stable currents (or stable varifolds) in a compact, simply-connected $\frac{1}{4}$-pinched Riemannian manifold.

There are several results supporting this conjecture (e.g., [Am], [Ho], [HW1], $[\mathrm{Ok}]$ ). Here we also give a partial positive answer to this conjecture on complete $\delta$-pinched hypersurfaces immersed in the Euclidean space $R^{n+1}$. Concretely, in the present paper we prove the following

Main theorem. Let $N(c)$ be a real space form with constant sectional curvature $c(\geq 0)$ and $M \hookrightarrow N(c)$ an $n(\geq 3)$-dimensional complete hypersurface immersed in $N(c)$. If the sectional curvature $K_{M}$ of $M$ satisfies the following pinching condition:

$$
c+\delta<K_{M} \leq c+1
$$

where $\delta=1 / 5$ for $n \geq 7, \delta=1 / 4$ for $n=5,6$ and $\delta=1 / 3$ for $n=3,4$, then there are no stable currents (or stable varifolds) in $M$.

Received by the editors March 15, 1999.

1991 Mathematics Subject Classification. Primary 53C42; Secondary 58A25.

Key words and phrases. Stable currents, $\delta$-pinched manifolds, $\delta$-pinched hypersurfaces.

This research was supported in part by NNSFC and NSFZP.

(C)2000 American Mathematical Society 
As a direct result of the theorem, we then have

Corollary. There are no stable currents (or stable varifolds) on an $n(\geq 3)$-dimensional complete $\delta$-pinched hypersurface of the Euclidean space $R^{n+1}$ where $\delta=1 / 5$ for $n \geq 7, \delta=1 / 4$ for $n=5,6$ and $\delta=1 / 3$ for $n=3,4$.

Remark. For a "harmonic version" of the above conjecture of Lawson and Simons, a similar result has been obtained in [HPS] and [HW2].

\section{§2. Preliminaries}

In this section we prepare some necessary formulas and propositions.

Let $M$ be a compact Riemannian manifold and let $\mathcal{H}^{p}$ denote the Hausdorff $p$-measure on $M$. An oriented $p$-rectifiable set is a pair $\mathcal{S}=(S, \xi)$, where $S$ is a $p$ rectifiable subset and $\xi: S \rightarrow \bigwedge^{p} T M$ is an $\mathcal{H}^{p}$-measurable section of $p$-th exterior product of the tangent bundle over $M$ satisfying the fact that for $\mathcal{H}^{p}$-almost all $x \in S, \xi_{x}$ is a simple vector of unit length which represents $T_{x} S$. Given a smooth p-form $\omega \in \bigwedge^{p} T^{*} M$, the integral

$$
\mathcal{S}(\omega)=\int_{S} \omega\left(\xi_{x}\right) d \mathcal{H}^{p}(x)
$$

defines $\mathcal{S}$ as a continuous linear functional on $\bigwedge^{p} T^{*} M$, so that $\mathcal{S}$ has a norm

$$
\mathbf{M}(\mathcal{S})=\mathcal{H}^{p}(S)
$$

Consider the following set:

$$
\mathcal{R}_{p}(M)=\left\{\mathcal{S}=\sum_{k=1}^{\infty} k \mathcal{S}_{k} \mid \mathcal{S}_{k}=\left(S_{k}, \xi_{k}\right), \mathbf{M}(\mathcal{S})=\sum k \mathcal{H}^{p}\left(S_{k}\right)<\infty\right\} .
$$

An element $\mathcal{S}$ of $\mathcal{R}_{p}(M)$ is called a rectifiable p-current with norm $\mathbf{M}(\mathcal{S})$.

For any vector field $V \in T M$, let $\phi_{t}: M \rightarrow M$ be the 1-parameter group of diffeomorphisms generated by $V$. Then for each $t$ we have the rectifiable current $\phi_{t}^{*}(\mathcal{S})$ defined as

$$
\phi_{t}^{*}(\mathcal{S})(\omega)=\mathcal{S}\left(\phi_{t}^{*} \omega\right)
$$

for $\omega \in \bigwedge^{p} T^{*} M$. A current $\mathcal{S} \in \mathcal{R}_{p}(M)$ is said to be stable if for each vector field $V$ there is an $\varepsilon>0$ such that

$$
\mathbf{M}\left(\phi_{t}^{*} \mathcal{S}\right) \geq \mathbf{M}(\mathcal{S})
$$

for $|t| \leq \varepsilon$. The first and second variational formulas for currents have been given by Lawson and Simons (see [LS] for details).

Let $N(c)$ be a real space form with constant sectional curvature $c \geq 0$, and let $M$ be a submanifold isometrically immersed in $N(c)$. Denote by $\bar{\nabla}$ and $\nabla$ the Levi-Civita connections of $N(c)$ and $M$, respectively. Then the second fundamental form of $M$ in $N(c)$ is defined as

$$
B(X, Y)=\bar{\nabla}_{X} Y-\nabla_{X} Y
$$


for $X, Y \in T M$, so that $B$ is a symmetric tensor field on $M$ with values in the cross section of normal bundle of $M$ in $N(c)$. The Gauss equation of $M$ is

$$
\begin{aligned}
R(X, Y, Z, W)= & c(\langle X, Z\rangle\langle Y, W\rangle-\langle X, W\rangle\langle Y, Z\rangle) \\
& +\langle B(X, Z), B(Y, W)\rangle-\langle B(X, W), B(Y, Z)\rangle
\end{aligned}
$$

for $X, Y, Z, W \in T M$, where $R$ denotes the curvature tensor of $M$. The following proposition is well known from [S.

Proposition 2.1. Let $N(c)$ be a real space form with constant sectional curvature $c(\geq 0)$ and $M \hookrightarrow N(c)$ an n-dimensional compact submanifold with the second fundamental form $B$ in $N(c)$. If at each point of $M$

$$
\sum_{r=p+1}^{n} \sum_{i=1}^{p}\left\{2\left\|B\left(e_{i}, e_{r}\right)\right\|^{2}-\left\langle B\left(e_{i}, e_{i}\right), B\left(e_{r}, e_{r}\right)\right\rangle\right\}<p(n-p) c
$$

for any local orthonormal frame field $\left\{e_{i}, e_{r}\right\}$ on $M$, where $0<p<n$, then there are no stable p-currents (or stable p-varifolds) in $M$. Moreover,

$$
H_{p}(M, \mathbb{Z})=H_{n-p}(M, \mathbb{Z})=0
$$

By using a straightforward estimate, we have from (2.4) and (2.5)

Corollary 2.2. Let $M$ be a n-dimensional complete $\delta$-pinched submanifold with the second fundamental form $B$ in the Euclidean space. If $\|B\|^{2} \leq 2 \delta(n-1+2 \sqrt{n-1})$ at each point of $M$, then there are no stable currents (or stable varifolds) in $M$.

Now let $M$ be an $n$-dimensional hypersurface with codimension 1 in $N(c)$. From now on we make use of the following convention on ranges of indices unless otherwise stated:

$$
1 \leq \alpha, \beta, \gamma, \cdots \leq n ; \quad 1 \leq i, j, \cdots \leq p ; \quad p+1 \leq r, s, \cdots \leq n
$$

Let $x \in M$ be an arbitrary point of $M$ and let $\left\{\lambda_{\alpha}\right\}$ be principal curvatures of $M$ corresponding to the principal direction vectors $\left\{\tilde{e}_{\alpha}\right\}$ which form an orthonormal basis at the point $x \in M$. Clearly, in such a basis, the second fundamental form of $M$ is diagonalized. From (2.4) it follows that

$$
R_{\alpha \beta \alpha \beta}=c+\lambda_{\alpha} \lambda_{\beta} \quad(\alpha \neq \beta)
$$

at $x \in M$, where $R_{\alpha \beta \alpha \beta}=R\left(\tilde{e}_{\alpha}, \tilde{e}_{\beta}, \tilde{e}_{\alpha}, \tilde{e}_{\beta}\right)$.

On putting

$$
e_{\alpha}=\sum_{\beta} a_{\alpha}^{\beta} \tilde{e}_{\beta}
$$


for a special orthogonal matrix $\left(a_{\alpha}^{\beta}\right)$, we have from $(2.5)$ and $(2.7)$

$$
\begin{aligned}
\sum_{r, i}\left\{2\left\|B\left(e_{i}, e_{r}\right)\right\|^{2}-\left\langle B\left(e_{i}, e_{i}\right), B\left(e_{r}, e_{r}\right)\right\rangle\right\} & \sum_{\alpha, \beta} \lambda_{\alpha} \lambda_{\beta}\left\{2\left(\sum_{i} a_{i}^{\alpha} a_{i}^{\beta}\right)\left(\sum_{r} a_{r}^{\alpha} a_{r}^{\beta}\right)-\sum_{i, r}\left(a_{i}^{\alpha}\right)^{2}\left(a_{r}^{\beta}\right)^{2}\right\} \\
= & \sum_{\alpha, i, r}\left(\lambda_{\alpha}\right)^{2}\left(a_{i}^{\alpha}\right)^{2}\left(a_{r}^{\alpha}\right)^{2} \\
& +\sum_{\alpha \neq \beta} \lambda_{\alpha} \lambda_{\beta}\left\{2\left(\sum_{i} a_{i}^{\alpha} a_{i}^{\beta}\right)\left(\sum_{r} a_{r}^{\alpha} a_{r}^{\beta}\right)-\sum_{i, r}\left(a_{i}^{\alpha}\right)^{2}\left(a_{r}^{\beta}\right)^{2}\right\} \\
= & \sum_{\alpha, i, r}\left(\lambda_{\alpha}\right)^{2}\left(a_{i}^{\alpha}\right)^{2}\left(a_{r}^{\alpha}\right)^{2}+\sum_{\alpha \neq \beta} \lambda_{\alpha} \lambda_{\beta} \\
& \cdot\left\{\left(\sum_{\gamma} a_{\gamma}^{\alpha} a_{\gamma}^{\beta}\right)^{2}-\left(\sum_{i} a_{i}^{\alpha} a_{i}^{\beta}\right)^{2}-\left(\sum_{r} a_{r}^{\alpha} a_{r}^{\beta}\right)^{2}-\sum_{i, r}\left(a_{i}^{\alpha}\right)^{2}\left(a_{r}^{\beta}\right)^{2}\right\} \\
= & \sum_{\alpha, i, r}\left(\lambda_{\alpha}\right)^{2}\left(a_{i}^{\alpha}\right)^{2}\left(a_{r}^{\alpha}\right)^{2} \\
& -\sum_{\alpha \neq \beta} \lambda_{\alpha} \lambda_{\beta}\left\{\left(\sum_{i} a_{i}^{\alpha} a_{i}^{\beta}\right)^{2}+\left(\sum_{r} a_{r}^{\alpha} a_{r}^{\beta}\right)^{2}+\sum_{i, r}\left(a_{i}^{\alpha}\right)^{2}\left(a_{r}^{\beta}\right)^{2}\right\},
\end{aligned}
$$

from which it follows that

$$
\sum_{r, i}\left\{2\left\|B\left(e_{i}, e_{r}\right)\right\|^{2}-\left\langle B\left(e_{i}, e_{i}\right), B\left(e_{r}, e_{r}\right)\right\rangle\right\}=F(p, n),
$$

where

$$
F(p, n) \equiv \sum_{\alpha, r, i}\left(\lambda_{\alpha}\right)^{2}\left(a_{i}^{\alpha}\right)^{2}\left(a_{r}^{\alpha}\right)^{2}-\frac{1}{2} \sum_{\alpha \neq \beta} \lambda_{\alpha} \lambda_{\beta} G(\alpha, \beta),
$$

$$
G(\alpha, \beta) \equiv 2\left(\sum_{i} a_{i}^{\alpha} a_{i}^{\beta}\right)^{2}+2\left(\sum_{r} a_{r}^{\alpha} a_{r}^{\beta}\right)^{2}+\sum_{r, i}\left[\left(a_{i}^{\alpha}\right)^{2}\left(a_{r}^{\beta}\right)^{2}+\left(a_{i}^{\beta}\right)^{2}\left(a_{r}^{\alpha}\right)^{2}\right] .
$$

Thus, by Proposition 2.1 we have immediately the following

Proposition 2.3. Let $N(c)$ be a real space form with constant sectional curvature $c(\geq 0)$ and $M \hookrightarrow N(c)$ an n-dimensional compact hypersurface with principal curvatures $\left\{\lambda_{\alpha}\right\}$ in $N(c)$. If at each point of $M$

$$
F(p, n)<p(n-p) c
$$

for any special orthogonal matrix $\left(a_{\alpha}^{\beta}\right)$, where $0<p<n$ and $F(p, n)$ is defined by (2.8), then there are no stable $p$-currents (or stable p-varifolds) in $M$. 


\section{§3. Some Lemmas}

We begin with the following algebraic lemma.

Lemma 3.1. Let $\left(a_{\alpha}^{\beta}\right)$ be a special orthogonal matrix. Then

$$
\sum_{i, r} a_{i}^{\alpha} a_{i}^{\beta} a_{r}^{\alpha} a_{r}^{\beta} \leq \frac{1}{4} \delta_{\beta}^{\alpha}
$$

Proof.

$$
\begin{aligned}
\sum_{i, r} a_{i}^{\alpha} a_{i}^{\beta} a_{r}^{\alpha} a_{r}^{\beta} & \leq \frac{1}{2}\left\{\left(\sum_{i} a_{i}^{\alpha} a_{i}^{\beta}\right)^{2}+\left(\sum_{r} a_{r}^{\alpha} a_{\mathrm{r}}^{\beta}\right)^{2}\right\} \\
& =\frac{1}{2}\left\{\delta_{\beta}^{\alpha}-2 \sum_{i, r} a_{i}^{\alpha} a_{i}^{\beta} a_{r}^{\alpha} a_{r}^{\beta}\right\},
\end{aligned}
$$

from which (3.1) follows directly.

Now suppose that the sectional curvature $K_{M}$ of $M$ satisfies the following pinching condition:

$$
c+\varepsilon^{2}<K_{M} \leq c+1
$$

for some positive number $\varepsilon<1$. (3.3) together with (2.6) yields that

$$
\varepsilon^{2}<\lambda_{\alpha} \lambda_{\beta} \leq 1 \quad(\alpha \neq \beta),
$$

which implies that all of $\left\{\lambda_{\alpha}\right\}$ are nonzero and have the same sign. So, without loss of generality, we may assume that at $x \in M$

$$
0<\lambda_{1} \leq \lambda_{2} \leq \cdots \leq \lambda_{n}
$$

By using (3.4) and (3.5), one can see the following statement is true.

Lemma 3.2. If (3.4) and (3.5) hold, then we have

(1) $\lambda_{\alpha}>\varepsilon$ for $\alpha \neq 1$;

(2) $\lambda_{\alpha} \leq 1$ for $\alpha \neq n$;

(3) $\lambda_{n}<\varepsilon^{-1}$ and $\lambda_{1}>\varepsilon^{2}$ if $n \geq 3$.

In the following, all calculations will be made out at a point $x \in M$ where (3.4) and (3.5) hold.

Lemma 3.3. If (3.4) and (3.5) hold, then we have

$$
F(p, n)<-p(n-p) \varepsilon^{2}+\sum_{\alpha, i, r}\left(\lambda_{\alpha}^{2}-\varepsilon^{2}\right)\left(a_{i}^{\alpha}\right)^{2}\left(a_{r}^{\alpha}\right)^{2},
$$

where $F(p, n)$ is defined by (2.8). 
Proof. By using (2.9) and the fact that the matrix $\left(a_{\alpha}^{\beta}\right)$ is a special orthogonal matrix, we have

$$
\begin{aligned}
\frac{1}{2} \sum_{\alpha, \beta} G(\alpha, \beta)= & \sum_{i, j}\left(\sum_{\alpha, \beta} a_{i}^{\alpha} a_{j}^{\alpha} a_{i}^{\beta} a_{j}^{\beta}\right)+\sum_{r, s}\left(\sum_{\alpha, \beta} a_{r}^{\alpha} a_{s}^{\alpha} a_{r}^{\beta} a_{s}^{\beta}\right) \\
& +\sum_{i, r}\left(\sum_{\alpha}\left(a_{i}^{\alpha}\right)^{2}\right)\left(\sum_{\beta}\left(a_{r}^{\beta}\right)^{2}\right) \\
= & n+p(n-p) .
\end{aligned}
$$

From this equation and (3.4) it follows that

$$
\begin{gathered}
-\frac{1}{2} \sum_{\alpha \neq \beta} \lambda_{\alpha} \lambda_{\beta} G(\alpha, \beta)<-\frac{1}{2} \varepsilon^{2} \sum_{\alpha, \beta} G(\alpha, \beta)+\frac{1}{2} \varepsilon^{2} \sum_{\alpha} G(\alpha, \alpha) \\
=-\varepsilon^{2}[n+p(n-p)]+\varepsilon^{2} \sum_{\alpha}\left\{1-\sum_{i, r}\left(a_{i}^{\alpha}\right)^{2}\left(a_{r}^{\alpha}\right)^{2}\right\} \\
=-p(n-p) \varepsilon^{2}-\varepsilon^{2} \sum_{i, r}\left(a_{i}^{\alpha}\right)^{2}\left(a_{r}^{\alpha}\right)^{2} .
\end{gathered}
$$

Inserting (3.7) into (2.8) yields (3.6).

Lemma 3.4. Under the same hypothesis in Lemma 3.3, we also have

$$
\begin{aligned}
F(p, n)< & -p(n-p) \varepsilon^{2}+\frac{1}{4} \sum_{\alpha \neq 1, n}\left(\lambda_{\alpha}^{2}-\varepsilon^{2}\right) \\
& +\frac{1}{4}\left(\lambda_{n}-\varepsilon\right)^{2}+\left(\lambda_{1}^{2}-\varepsilon^{2}\right) \sum_{i, r}\left(a_{i}^{1}\right)^{2}\left(a_{r}^{1}\right)^{2} \\
& -\varepsilon\left(\lambda_{n}-\varepsilon\right)\left\{(n-p) \sum_{i}\left(a_{i}^{n}\right)^{2}+p \sum_{r}\left(a_{r}^{n}\right)^{2}\right\} \\
& -\left(\lambda_{1}-\varepsilon\right) \lambda_{n} G(1, n),
\end{aligned}
$$

where $G(1, n)$ is defined by $(2.9)$.

Proof. By using (3.4) and the property (1) in Lemma 3.2, we have

$$
\begin{aligned}
-\frac{1}{2} \sum_{\alpha \neq \beta} \lambda_{\alpha} \lambda_{\beta} G(\alpha, \beta)< & -\frac{1}{2} \varepsilon^{2} \sum_{\alpha, \beta \neq n} \sum_{\alpha \neq \beta} G(\alpha, \beta)-\varepsilon \lambda_{n} \sum_{\alpha \neq 1, n} G(\alpha, n) \\
& -\lambda_{1} \lambda_{n} G(1, n) \\
= & -\frac{1}{2} \varepsilon^{2} \sum_{\alpha \neq \beta} G(\alpha, \beta)-\varepsilon\left(\lambda_{n}-\varepsilon\right) \sum_{\alpha \neq n} G(\alpha, n) \\
& -\left(\lambda_{1}-\varepsilon\right) \lambda_{n} G(1, n),
\end{aligned}
$$


where $G(\alpha, \beta)$ is defined by (2.9). By substituting (3.9) into (2.8) and using (3.7), we can get

$$
\begin{aligned}
F(p, n)< & -p(n-p) \varepsilon^{2}+\sum_{\alpha, i, r}\left(\lambda_{\alpha}^{2}-\varepsilon^{2}\right)\left(a_{i}^{\alpha}\right)^{2}\left(a_{r}^{\alpha}\right)^{2} \\
& -\varepsilon\left(\lambda_{n}-\varepsilon\right) \sum_{\alpha \neq n} G(\alpha, n)-\left(\lambda_{1}-\varepsilon\right) \lambda_{n} G(1, n) .
\end{aligned}
$$

Since the matrix $\left(a_{\alpha}^{\beta}\right)$ is a special orthogonal matrix, then we see easily that

$$
\begin{aligned}
\sum_{\alpha \neq n} G(\alpha, n)= & 2 \sum_{i, j} a_{i}^{n} a_{j}^{n}\left(\delta_{j}^{i}-a_{i}^{n} a_{j}^{n}\right)+2 \sum_{r, s} a_{r}^{n} a_{s}^{n}\left(\delta_{s}^{r}-a_{r}^{n} a_{s}^{n}\right) \\
& +\sum_{i, r}\left(a_{r}^{n}\right)^{2}\left[1-\left(a_{i}^{n}\right)^{2}\right]+\sum_{i, r}\left(a_{i}^{n}\right)^{2}\left[1-\left(a_{r}^{n}\right)^{2}\right] \\
= & 2 \sum_{i, r}\left(a_{i}^{n}\right)^{2}\left(a_{r}^{n}\right)^{2}+(n-p) \sum_{i}\left(a_{i}^{n}\right)^{2}+p \sum_{r}\left(a_{r}^{n}\right)^{2} .
\end{aligned}
$$

By inserting (3.11) into (3.10), we have

$$
\begin{aligned}
F(p, n)< & -p(n-p) \varepsilon^{2}+\sum_{\alpha \neq 1, n}\left(\lambda_{\alpha}^{2}-\varepsilon^{2}\right)\left[\sum_{i, r}\left(a_{i}^{\alpha}\right)^{2}\left(a_{r}^{\alpha}\right)^{2}\right] \\
& +\left(\lambda_{1}^{2}-\varepsilon^{2}\right) \sum_{i, r}\left(a_{i}^{1}\right)^{2}\left(a_{r}^{1}\right)^{2}+\left(\lambda_{n}-\varepsilon\right)^{2} \sum_{i, r}\left(a_{i}^{n}\right)^{2}\left(a_{r}^{n}\right)^{2} \\
& -\varepsilon\left(\lambda_{n}-\varepsilon\right)\left\{(n-p) \sum_{i}\left(a_{i}^{n}\right)^{2}+p \sum_{r}\left(a_{r}^{n}\right)^{2}\right\}-\left(\lambda_{1}-\varepsilon\right) \lambda_{n} G(1, n) .
\end{aligned}
$$

By noting (1) of Lemma 3.2 and using Lemma 3.1 with $\alpha=\beta$, now (3.8) follows from the above inequality directly.

\section{$\S 4$. Proof of the Main theOREM}

First of all, the pinching condition for the curvature in the theorem implies that $M$ is compact by the theorem of Bonnet-Meyers. By Proposition 2.3, it is sufficient to prove that $F(p, n)<0$ for any $0<p<n$, where $F(p, n)$ is defined by (2.8), because $c \geq 0$.

For a fixed point $x \in M$, the same notations in $\S 2$ and $\S 3$ will be adopted and all calculations will be carried out at that point $x$. The proof is separated into two steps.

The first step. Suppose that $\lambda_{1} \geq \varepsilon$.

Since $p(n-p) \geq n-1$ for any $1 \leq p \leq n-1$ and $G(1, n)$ defined by $(2.9)$ is nonnegative, then we have from (3.8)

$$
\begin{aligned}
F(p, n)< & -(n-1) \varepsilon^{2}+\frac{1}{4} \sum_{\alpha \neq n}\left(\lambda_{\alpha}^{2}-\varepsilon^{2}\right)+\frac{1}{4}\left(\lambda_{n}-\varepsilon\right)^{2} \\
& -\varepsilon\left(\lambda_{n}-\varepsilon\right)\left\{(n-p) \sum_{i}\left(a_{i}^{n}\right)^{2}+p \sum_{r}\left(a_{r}^{n}\right)^{2}\right\},
\end{aligned}
$$

where Lemma 3.1 has been used for $\alpha=\beta=1$. 
By virtue of (1) and (2) in Lemma 3.2 and noting that

$$
(n-p) \sum_{i}\left(a_{i}^{n}\right)^{2}+p \sum_{r}\left(a_{r}^{n}\right)^{2} \geq \sum_{\alpha}\left(a_{\alpha}^{n}\right)^{2}=1,
$$

(4.1) may be reduced to

$$
\begin{aligned}
F(p, n) & <-(n-1) \varepsilon^{2}+\frac{n-1}{4}\left(1-\varepsilon^{2}\right)+\frac{1}{4}\left(\lambda_{n}-\varepsilon\right)^{2}-\varepsilon\left(\lambda_{n}-\varepsilon\right) \\
& =\frac{1}{4}\left\{(n-1)\left(1-5 \varepsilon^{2}\right)+\left(\lambda_{n}-\varepsilon\right)\left(\lambda_{n}-5 \varepsilon\right)\right\} .
\end{aligned}
$$

From this equation, together with (3) of Lemma 3.2, it follows that

$$
\begin{aligned}
F(p, n) & <\frac{1}{4}\left\{(n-1)\left(1-5 \varepsilon^{2}\right)+\left(\lambda_{n}-\varepsilon\right)\left(\varepsilon^{-1}-5 \varepsilon\right)\right\} \\
& =-\frac{1}{4}\left(5 \varepsilon^{2}-1\right)\left[n-1+\varepsilon^{-1}\left(\lambda_{n}-\varepsilon\right)\right] \leq 0
\end{aligned}
$$

for $\varepsilon^{2}=\delta \geq 1 / 5$. Thus, under the pinching condition in the theorem, (4.2) holds for any $0<p<n$. Hence, the theorem follows from Proposition 2.3 directly.

The second step. Suppose that $\lambda_{1}<\varepsilon$.

Since $\varepsilon<\lambda_{n}<\varepsilon^{-1}$ according to (1) and (3) in Lemma 3.2, we consider two cases separately.

Case (i). $\varepsilon<\lambda_{n} \leq 1$.

From Lemma 3.3 it follows that

$$
F(p, n)<-(n-1) \varepsilon^{2}+\sum_{\alpha \neq 1}\left(\lambda_{\alpha}^{2}-\varepsilon^{2}\right) \sum_{i, r}\left(a_{i}^{\alpha}\right)^{2}\left(a_{r}^{\alpha}\right)^{2},
$$

which together with (3.1) and (2) of Lemma 3.2 yields

$$
\begin{aligned}
F(p, n) & <-(n-1) \varepsilon^{2}+\frac{1}{4} \sum_{\alpha \neq 1}\left(\lambda_{\alpha}^{2}-\varepsilon^{2}\right) \\
& \leq-(n-1) \varepsilon^{2}+\frac{1}{4}(n-1)\left(1-\varepsilon^{2}\right) \\
& =\frac{n-1}{4}\left(1-5 \varepsilon^{2}\right) \leq 0
\end{aligned}
$$

for $\varepsilon^{2}=\delta \geq 1 / 5$. By the same reason as in the first step, the theorem follows.

Case (ii). $1<\lambda_{n}<\varepsilon^{-1}$.

By Lemma 3.3 and Lemma 3.1, we have

$$
\begin{aligned}
F(p, n) & <-(n-1) \varepsilon^{2}+\sum_{\alpha \neq 1}\left(\lambda_{\alpha}^{2}-\varepsilon^{2}\right) \sum_{i, r}\left(a_{i}^{\alpha}\right)^{2}\left(a_{r}^{\alpha}\right)^{2} \\
& \leq-(n-1) \varepsilon^{2}+\frac{1}{4} \sum_{\alpha \neq 1}\left(\lambda_{\alpha}^{2}-\varepsilon^{2}\right) .
\end{aligned}
$$

By means of (3.4), we have $\lambda_{\alpha} \leq 1 / \lambda_{n}$ for $\alpha \neq n$. So, (4.4) can be reduced as

$$
\begin{aligned}
F(p, n) & <-(n-1) \varepsilon^{2}+\frac{n-2}{4}\left(\lambda_{n}^{-2}-\varepsilon^{2}\right)+\frac{1}{4}\left(\lambda_{n}^{2}-\varepsilon^{2}\right) \\
& =\frac{1}{4 \lambda_{n}^{2}}\left\{\lambda_{n}^{4}-5(n-1) \varepsilon^{2} \lambda_{n}^{2}+n-2\right\} .
\end{aligned}
$$


Assume that $\varepsilon^{2}=\delta=1 / 5$. An element estimate gives

$$
\lambda_{n}^{4}-(n-1) \lambda_{n}^{2}+n-2 \leq 0 \quad \text { for } \quad n \geq 7 \quad \text { and } \quad 1<\lambda_{n}^{2}<5 .
$$

Assume that $\varepsilon^{2}=\delta=1 / 4$. A similar reason gives

$$
\lambda_{n}^{4}-\frac{5}{4}(n-1) \lambda_{n}^{2}+n-2 \leq 0 \quad \text { for } n \geq 5 \quad \text { and } 1<\lambda_{n}^{2}<4 .
$$

Assume that $\varepsilon^{2}=\delta=1 / 3$. A similar reason gives

$$
\lambda_{n}^{4}-\frac{5}{3}(n-1) \lambda_{n}^{2}+n-2 \leq 0 \quad \text { for } n \geq 3 \quad \text { and } 1<\lambda_{n}^{2}<3 .
$$

Hence, under the hypothesis in the theorem, we have from $(4.5)$ that $F(p, n)<0$ for any $0<p<n$, which together with Proposition 2.3 implies that the theorem holds.

In summary, the main theorem is proved completely because the point $x \in M$ is arbitrary.

\section{$\S 5$. Examples}

Let $R^{n+1}$ be the Euclidean $(n+1)$-space with Cartesian coordinates $x_{1}, \cdots, x_{n+1}$. Consider the following ellipsoid

$$
\Theta^{n}=\left\{\left(x_{1}, \cdots, x_{n+1}\right) \in R^{n+1}: \frac{x_{1}^{2}}{a_{1}^{2}}+\cdots+\frac{x_{n+1}^{2}}{a_{n+1}^{2}}=1\right\}
$$

with $0<a_{1} \leq a_{2} \leq \cdots \leq a_{n+1}$. By $[\mathrm{SP}$, the minimal and maximal principal curvatures of $\Theta^{n}$ in $R^{n+1}$ are respectively

$$
\lambda_{1}=a_{1} / a_{n+1}^{2} \quad \text { and } \quad \lambda_{n}=a_{n+1} / a_{1}^{2} .
$$

Thus, by the Gauss equation, the sectional curvature $K_{\Theta}$ of $\Theta^{n}$ satisfies

$$
\frac{a_{1}^{2}}{a_{n+1}^{4}} \leq K_{\Theta} \leq \frac{a_{n+1}^{2}}{a_{1}^{4}}
$$

Hence, $\Theta^{n}$ is $\delta$-pinched if

$$
\left(a_{1} / a_{n+1}\right)^{6} \geq \delta
$$

By the corollary, we have the following

Example 1. Let $\Theta^{n}(n \geq 3)$ be an ellipsoid in $R^{n+1}$ defined by (5.1) satisfying (5.4), where $\delta=1 / 5$ for $n \geq 7, \delta=1 / 4$ for $n \geq 5$ and $\delta=1 / 3$ for $n \geq 3$. Then, there are no stable currents (or stable varifolds) on $\Theta^{n}$.

We now consider the case of $n=2$. In such a case, (2.7) can be replaced by

$$
\begin{gathered}
e_{1}=\cos \theta \tilde{e}_{1}+\sin \theta \tilde{e}_{2}, \\
e_{2}=-\sin \theta \tilde{e}_{1}+\cos \theta \tilde{e}_{2},
\end{gathered}
$$

for some $\theta \in[0,2 \pi)$. Clearly, (2.8) with $p=1$ yields that

$$
\begin{gathered}
F(1,2)=\frac{1}{4}(\sin 2 \theta)^{2}\left(\lambda_{2}-\lambda_{1}\right)^{2}-\lambda_{1} \lambda_{2} \\
\leq \frac{\lambda_{2}^{2}}{4}\left\{\left(\frac{\lambda_{1}}{\lambda_{2}}\right)^{2}-6\left(\frac{\lambda_{1}}{\lambda_{2}}\right)+1\right\} .
\end{gathered}
$$


Thus, $F(1,2)<0$ if

$$
3+2 \sqrt{2}>\left(\lambda_{1} / \lambda_{2}\right)>3-2 \sqrt{2} .
$$

By Proposition 2.3, we have

Example 2. Let $M^{2}$ be an ovaloid in $R^{3}$ with principal curvatures $\lambda_{1} \leq \lambda_{2}$ satisfying (5.7). Then there are no any stable closed geodesics on $M^{2}$.

\section{REFERENCES}

[Am] Aminov,J., On the instability of a minimal surface in an $N$-dimensional Riemannian space of positive curvature, Math. USSR Sb., 29 (1976), 359-375.

[Ho] Howard,R., The nonexistence of stable submanifolds, varifolds, and harmonic maps in sufficiently pinched simply connected Riemannian manifolds, Michigan Math. J., 32 (1985), 321-334. MR 87h:58040

[HW1] Howard,R. and Wei,S.W., On the existence and non-existence of stable submanifolds and currents in positively curved manifolds and the topology of submanifolds, preprint.

[HW2] _ Non-existence of stable harmonic maps to and from certain homogeneous spaces and submanifolds of Euclidean space, Trans. Amer. Math. Soc., 294 (1986), 319-331. MR 87c:58033

[HPS] Hu,H.S., Pan,Y.L. and Shen,Y.B., On harmonic maps and a pinching theorem for positively curved hypersurfaces, Proc. Amer. Math. Soc., 99 (1987), 182-186. MR 87m:58042

[LS] Lawson,H.B. and Simons,J., On stable currents and their applications to global problems in real and complex geometry, Ann. of Math., 98 (1973), 427-450. MR 48:2881

[Ok] Okayasu,T., On the instability of minimal submanifolds in Riemannian manifolds of positive curvature, Math. Z., 201 (1989), 33-44. MR 90c:53158

[SP] Shen,Y.B. and Pan,Y.L., On harmonic maps from ellipsoids, Acta Math. Scientia, 6 (1986), 71-75 (Chinese).

Department of Mathematics, West-Brook Campus, Zhejiang University, Hangzhou 310028, People's Republic of China

E-mail address: ybshen@dial.zju.edu.cn 